\title{
Pattern of occurrence of obsessive-compulsive symptoms in Bipolar Disorder
}

Margherita Trinchieria, Daniel Benjamin Daniel ${ }^{b}$, Andrea Amerioc, Valeria Lucarinia, Martina Ferraria, Paolo Ossola ${ }^{a}$, Chiara De Panfilis ${ }^{\text {ab }}$, Carlo Marchesiab and Matteo Tonna. ${ }^{\text {b }}$

a Department of Neuroscience, Psychiatric Unit, University of Parma, Italy b Department of Mental Health, Local Health Service, Parma, Italy c Inpatient Mental Health Service, SS. Antonio e Biagio e Cesare Arrigo Hospital, Alessandria, Italy

\section{OBJECTIVE}

Apparent comorbidity between Bipolar Disorder (BD) and Obsessive-Compulsive Disorder (OCD) is a common condition, but the meaning of this comorbidity has not been clarified yet.

This study aimed to evaluate the pattern of occurrence of obsessive-compulsive symptoms (OCS) in Bipolar Disorder, particularly if OCS cycle with affective phases or represent an independent phenomenon.

\section{METHODS}

168 patients affected by Bipolar Disorder were assessed with the Yale-Brown Obsessive-Compulsive Scale (YBOCS), the Hamilton Depression Rating Scale (HAM-D) and the Young Mania Rating Scale (YMRS). Patients were divided into 4 groups according to the affective phase at the time of assessment: euthymic, manic, depressive and mixed episode.

\section{RESULTS}

\section{Severity of OCS (YBOCS total score) was} associated with depressive symptoms in the whole sample.

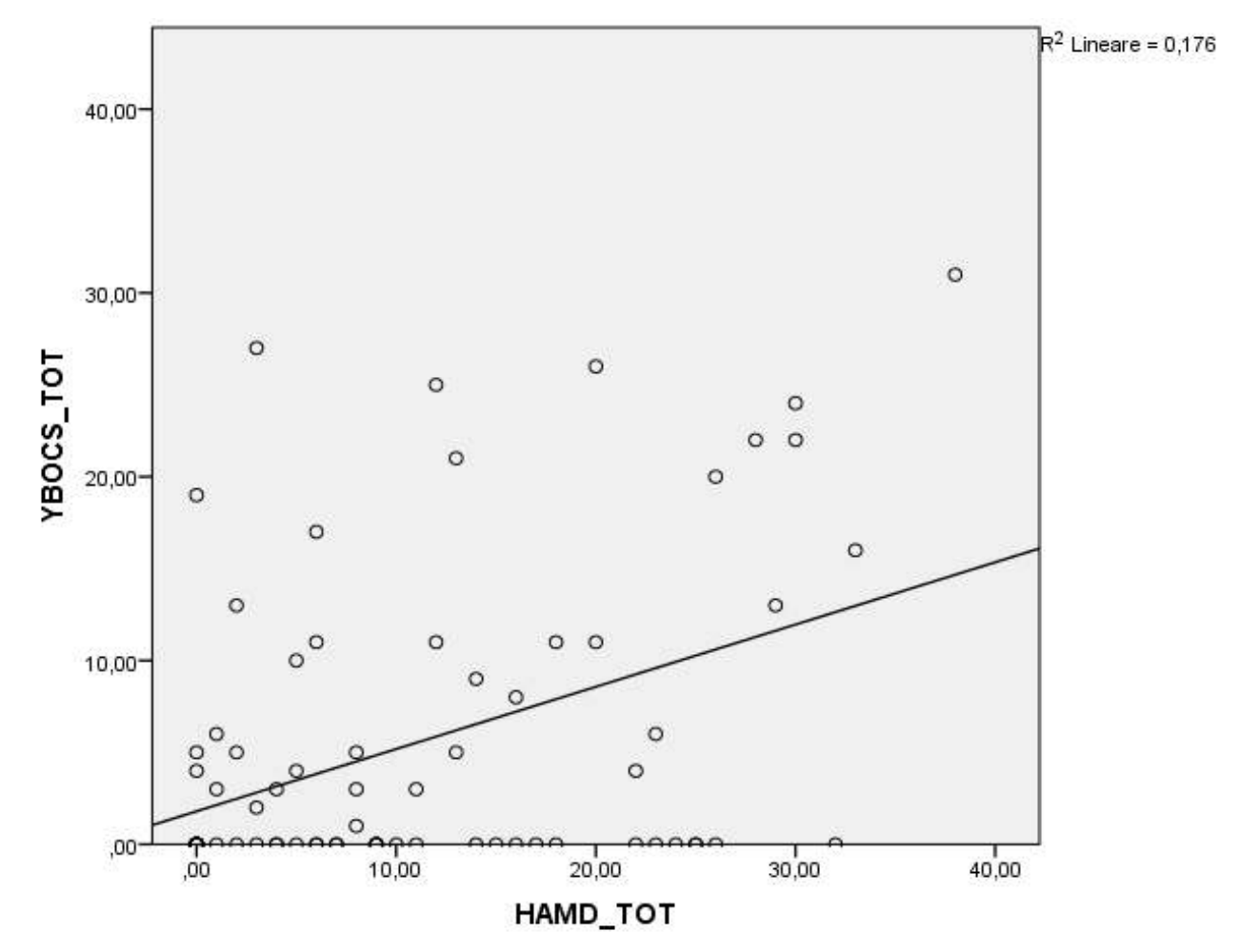

This relationship was confirmed during mixed episodes and manic phases. The highest severity of OCS was found in mixed episodes. YBOCS_TOT

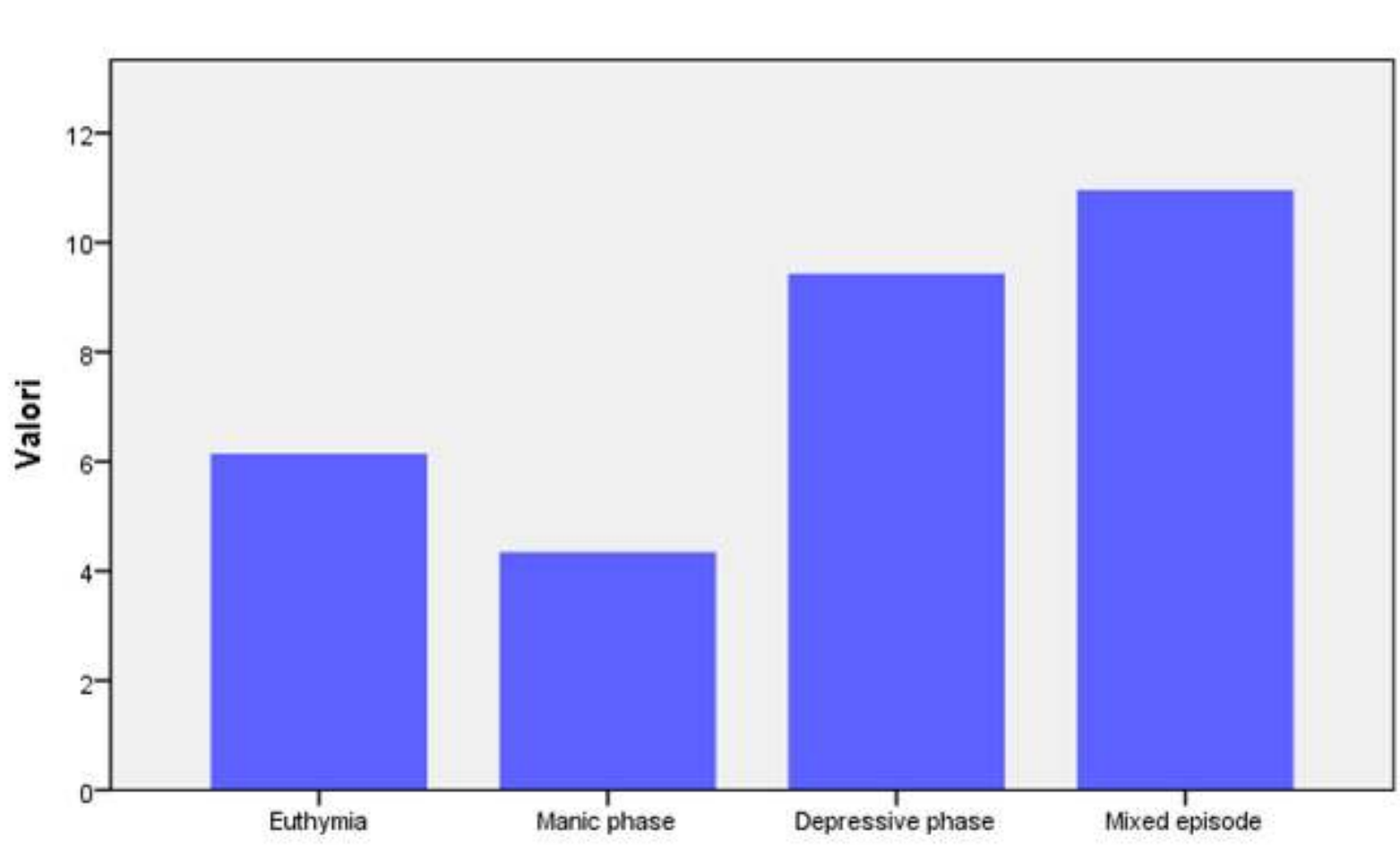

CONCLUSIONS

Our findings suggest that OCD-BD comorbidity does not represent the co-occurrence of two independent diseases.

Rather, OCS appear as a state-dependent phenomenon cycling with affective phases, i.e. worsening during depression and mixed states and improving during maniac phases. Since the strong relationship between OCS and depressive severity as well as the highest severity of OCS in mixed episodes, we hypothesize that OCS tend to exacerbate in the presence of depressive symptoms associated with hypomanic thought activation. 